\title{
The Intertwining of Disciplinary Concepts between Health Sciences and Economics
}

\author{
Mohsin Ahmad Khan ${ }^{1} \&$ John Whalley ${ }^{1,2,3}$ \\ ${ }^{1}$ Western University, London, Canada \\ ${ }^{2}$ Centre for International Governance and Innovation (CIGI), Waterloo, Canada \\ ${ }^{3}$ NBER, Cambridge, United States \\ Correspondence: John Whalley, Department of Economics, Western University, London, ON., N6A 5C2, Canada. \\ Tel: 1-519-661-2111 X 85243. E-mail: jwhalley@uwo.ca
}

Received: April 20, 2013 Accepted: May 13, 2013 Available online: September 22, 2013

doi:10.11114/ijsss.v1i2.131 URL: http://dx.doi.org/10.11114/ijsss.v1i2.131

\begin{abstract}
This paper focuses on the cross usage of terminology originating in one discipline but used in another, using economics and health sciences as our example. We highlight the use by economists of such terms as the brain, depression, and exhaustion whereas currency, elasticity, equilibrium, and optimality are all central notions in economics now being used in health sciences. We suggest that the dual usage occurs in ways where the original use and meaning of terms is not fully mirrored in their use in the other discipline. In part, this may reflect the pressures on researchers in all disciplines to be novel and innovative, and hence the incentive to adopt out of discipline terminology without full appreciation of its full meaning elsewhere. We discuss possible explanations for this characteristic of cross disciplinary use of terminology.
\end{abstract}

Keywords: interdisciplinarity, pair-wise comparison, depression, exhaustion, elasticity, equilibrium

\section{Introduction}

In an era in which pressures are being brought to bear by disciplinary research councils on individual researchers to move in an interdisciplinary direction, we discuss the growing joint use of concepts and terminology from specific disciplines in each other's discourse. We focus on health sciences and economics as a concrete pair-wise example, discussing what we label as terminological intertwining. Economics and health sciences are chosen as pairs for a number of reasons. One is the widespread suggestion that in light of the 2008 financial crises and the seeming inability of economists to first forecast and then adequately treat the problem, medicine based treatments for the economy should now be contemplated. Another is the growth of the sub-field of health economics within economics which is primarily concerned with social arrangements for health care delivery (insurance, patient-doctor relationships) and the impacts of health on economic performance (and vice versa). There has, however, been little discussion of the direct use of economic analysis in medical analysis of bodily function, or conversely the use of medical treatments for the economy which is what a more direct interdisciplinarity might suggest.

We illustrate our discussion using several instances of dual use of terminology and concepts in both economics and health sciences. We begin with economics and Stiglitz (2008), who has suggested that in light of first the 1997-1998 Asian financial crises and then the 2008 financial crisis that the key role of financial institutions suggests new thinking on banks as constituting the "brains of the economy", and those economists should modify their earlier analysis. Another is economist's diagnosis of an economy being in a depression, noting that economic depression differs from medically diagnosed depressive conditions. Yet another is in sports medicine, where "optimal performance/health" is a widely held objective; the concept of optimality seems to differ from that in economics. A further example was the 1930's talk of economic exhaustion, yet again the use of terminology differs greatly from the perspective of health sciences.

From the standpoint of health sciences, Adenosine Tri-phosphate (ATP), a universal energy carrier in the cells is often termed as molecular entity of "currency" of energy transfer within cells for metabolism, which hereby 
varies sharply from the definition of the word currency in the discipline of economics (Mcmillan, 2009). Further, there exists an example of parallel use of the terms "equilibrium" and "homeostasis" as central and key concept which seemingly refer to similar notions, but are interpreted differently in their subject of study. We also discuss the dual disciplinary use of "elasticity" and other concepts.

What seems to emerge is the implicit use of interdisciplinary conceptualization across the two communities of medical sciences and economics researchers and practitioners, but with the use of the concepts seemingly occurring in ways which often differ from the original use of concept in the other discipline. In some cases such concepts appear to be employed or adopted seemingly without a clear recognition or understanding of their intended initial meaning in the other discipline. This may perhaps reflect their use in some cases within discipline discourse, where proponents in debate seek to offer counter analyses to challenge positions of others within the discipline as much as to advance communication across disciplines more widely by injecting new terminology. We qualify this as a broad generalization by citing a number of instances where closer common disciplinary adoption of terminology also seems to occur.

Whether our characterization of economics/health sciences conceptual inter-twining transfers directly to other pair-wise disciplinary interactions we leave as a topic for future research. We do not find similar discussion in literature of other pair-wise disciplinary use of concepts, and emphasize that our evaluation of interdisciplinary intertwining is only one of many possible pair-wise analyses that are possible of emerging interdisciplinary interactions since many other pairs could be analyzed. At issue is how far the pressures within the academic community to strive for interdisciplinarity, with the benefits of improved information flow and fresh scholarly stimulus, may also have the negative side effect of fostering inconsistent use of concepts.

\section{Examples of Use of Medical Terminology in Economics}

We begin our discussion of the intertwining of disciplinary terminology by focusing on some examples of uses and analyses of medical terminology by economists. There seem to be a number of these, and our sense is that use of medical terminology in economics would appear to be growing. In part, and as we note in the introduction, this is a reflection of the view outside of economics of the seeming inability of the discipline to predict and later fully respond to the 2008 financial crisis. Among other things, this perception has provoked suggestions that we should view the economy in medical terms rather than in more conventional neoclassical optimizing format and modify policy prescriptions accordingly by treating symptoms rather than probe for deeper causes.

\subsection{Brains of the Economy versus Brains in Medical Sciences}

We begin with repeated suggestion by Nobel laureate Joseph Stiglitz that banks and financial institutions in general need to be viewed as the "brains" of the economy and our existing economic analysis of financial institutions needs to be appropriately modified. These analogies to brains when discussing the functioning of financial institutions began with Stiglitz's earlier analysis of the impacts of the Asian financial crisis of 1997/1998 on lower income countries such as Indonesia. Stiglitz had been Chief Economist at the World Bank and was critical of the policy stance of the IMF at the time who argued for fiscal conservatism and deficit reduction in affected Asian economies.

In analyzing the impacts of the crisis and discussing the appropriate policy response, Sliglitz argued that economists had to think of the impacts of the crisis in non-conventional terms, and argued that the role of banks in each economy was key and central. Most prior analyses by economists treated the financial sector as analogous to other sectors in producing something useful to other producers and consumers (financial intermediation services), but that was the limit of any discussion of a special role for banks in the economy. Pre-Stiglitz financial institutions were considered to be conceptually to be no different than producers of other goods and services. Stiglitz then later drew on his analogies in discussion of the 1997-1998 Asian Financial crisis, and again in later debate on appropriate response to the 2008 financial crisis.

Sliglitz (2008) put his argument in the following way “...the financial system can be compared to the brains of the economy. It allocates scarce capital among competing uses by trying to direct it to where it is more effective; in other words, where it yields the highest returns. The financial system also monitors the funds to ensure that they are used in the way promised. If the financial system breaks down, firms cannot get the working capital they need to continue existing levels of production..." In essence, Stiglitz argued that banks and their services should be viewed as essential to economies as central processors of information, not as just producers of one of many goods and services.

Development of this argument in more concrete medical terms would focus on medical literature in which the brain is regarded as one of the most complex and least understood organs of the human body. Analogous to the 
purpose of banks in Stiglitz's opinion, brain is seen as the decision center of the entire body responsible for vital regulatory functions such as temperature maintenance, regulating flow of blood and oxygen, as well as maintaining homeostasis, senses and reflexes. But it is much more. As the center of the nervous system in the body, in evolutionary terms, the brain is the extension of the spinal cord. It consists of gray matter (40\%) and white matter (60\%) contained within the skull with neurons and glial cells as its basic constituent, supporting metabolism, insulation and structural development. Overall, this critical organ can be perceived as a biological processor which is unique in its form and function to its counterfeit.

Anatomically, brain can be thought of as divided into three distinct parts. The lower section, the brain stem, consists of the medulla (which controls digestion, heart rate, breathing, other sensory and motor functions), and the cerebellum (responsible for coordinating muscle movement and senses). The second section, the mid-brain, is larger than the lower vertebra, which houses the thalamus (controlling information relay) and hypothalamus, which forms part of the limbic system which is responsible for hormone and emotion regulation. The hippocampus is part of the hypothalamus and is responsible for memory and navigation. Much of the lower and mid brain comprises systems which are capable of registering experiences and regulating behaviour largely outside of any conscious awareness. In essence, the human brain is a multilayered site with the outer layer composed of the most recent brain structure, and the deeper layers consisting of structures reflecting our shared evolutionary history with reptiles and mammals (Hoyle et al., 2005).

Finally, there is the third section, the forebrain, which appears as a bump in the brain of a frog and is enlarged to the cerebrum of higher life forms in humans and covers the brain stem like the head of a mushroom. It has further evolved in humans into the walnut-like configuration of left and right hemispheres. The highly convoluted surface of the hemispheres - the cortex - is about two millimetres thick and has a total surface area of about 1.5 square-meters. The structure of the cortex is complicated but it is here that most of the "high-level" functions associated with the mind are implemented. Some of its regions are highly specialized - for example, the occipital lobes located near the rear of the brain are associated with the visual system and the motor cortex helps coordinate all voluntary muscle movements. These multilevel functions of the brain find no analogy in economists' discussion of how banks function in the economy.

These functions also interact in different way is different individuals. In most people, the left hemisphere is dominant over the right in deciding on responses. In individuals with normal hemispheric dominance, the left hemisphere, which manages the right side of the body, controls language and general cognitive functions. The right hemisphere, controlling the left half of the body, manages nonverbal processes, such as attention, pattern recognition, line orientation and the detection of complex auditory tones. Although the two hemispheres are in continuous communication with each other, acting as independent parallel processors with complementary functions, the dominant left-hemisphere appears most closely associated with the conscious self.

Although the brain comprises just $2 \%$ of the total body weight (roughly 3 pounds), it consumes $20 \%$ of its oxygen supply and $25 \%$ of total body energy utilization and every year researchers continue to find new functions and ever more complex interactions. In the sub-area of neuro-plasticity, researchers have discovered how the brain has the ability to adapt to its environment, even after adulthood and how it can cope with changing circumstances. For instance, if a certain region of the brain is damaged due to a stroke, another area may take over some of its function challenging the belief that specific brain function is fixed and confined to certain regions.

The analogy of the brain to financial institutions in economics seemingly embodies few or none of the multi-faceted functions of brain physiology. And seemingly, the use of brain like analyses in economics could usefully explore whether such features are present in entities such as banks. Indeed, if the essential argument is one of necessity of financial institutions for economic activity one could seemingly equally well claim analogies of financial institutions to heart, kidneys or blood supply to other organs. Medical literature stresses many additional features of brain function besides the coordination and decision-making features which a medical economic analysis of banks might suggest. A major theme in health sciences literature is both this adaptability of the brain and its ability to orchestrate adaptability throughout the body. The analogy of banks to brains would thus seem to be able to be potentially enriched by an exploration of the possible applicability of these other brain functions to banking.

\subsection{Depression in Economics versus Medical Sciences}

Another medical term underlying economics is depression. The use of the term depression in economics clearly draws on medical antecedents, and accordingly to Wikipedia, it refers to "a sustained, long-term down-turn in economic activity in one or more economies." This is also the approach taken in basic macro economics 
textbooks. A depression is seen as a more severe downturn than a recession which is seen by economists as part of a normal business cycle. Hence, to economists, economic depressions are seen as essentially unusually severe recessions which are much longer than mild downturns, and accompanied by increases in unemployment and falls in output (see Krugman, 2009).

The depression of 1929-1933 is usually characterized as the "Great Depression" due to its severity, although previous depressions (such as 1816-1819 in the US) may well have been more severe. The contraction in output in 1929 to 1933 may have been 50\% in North America and Central Europe, while estimates for 1816-1819 in the U.S. are as high as $75 \%$.

Interestingly, the term depression is relatively recent in its now common usage of characterizing wakened economic performance. US president Munro is often credited with the first use of the term to describe the 1824 downturn in the US, although through the $19^{\text {th }}$ and early $20^{\text {th }}$ century the more widely used terms were fluctuations and crises. Herbert Hoover reintroduced the term into the debate on how to respond to the 1929 sharp downturn on the basis that it was a more neutral term than recession. It later became associated with the famous US economist Irving Fisher after his 1932 book on "Booms and Depressions."

In medical usage, however, depression is a general term used to capture a range of conditions that result in loss of normal activities of daily living due to a combination of genetic, biochemical and environmental conditions. Discussion of alternative remedies follows medical diagnosis and has lead to the emergences of treatments based on regression theories, but these treatments do not find parallels in proposed economic policy responses to economic depressions. In contrast to its medical use, the use of the term depression in economics remains relatively simple with no complex diagnostics or classification of many types, sub-types and forms of depressions (unlike business cycles in general).

In medical usage, according to the National Institute of Mental Health (NIMH, 2005), one of the most important categorizations of depressions is between major depressive disorders and Dysthymic disorder. Major depressive disorder, also called major depression, is thought to be characterized by a combination of symptoms that interfere with a person's ability to work, sleep, study, eat, and enjoy once pleasurable activities. Major depression is disabling and prevents a person from functioning normally. An episode of major depression may occur only once in a person's lifetime, but more often it recurs throughout a person's life. Dysthymic disorder, also called dysthymia, is a long-term (two year or longer) condition with less severe symptoms which may not disable a person but can prevent them from functioning normally or feeling well. People with dysthymia may also experience one or more episodes of major depression during their lifetimes.

Depressive disorders exhibit slightly different characteristics than those described above, or they may develop under special circumstances. Not all researchers agree on how to characterize and define these forms of depression. They include psychotic depression, which occurs when a severe depressive illness is accompanied by some form of psychosis, such as a break with reality, hallucinations, and delusions. They also include postpartum depression which diagnosed when a new mother develops a major depressive episode within one month after delivery. It is estimated that 10 to 15 percent of women experience postpartum depression after giving birth. Also, seasonal affective disorder (SAD), which is characterized by onset of depressive illness during the winter months, when there is less natural sunlight. This depression generally lifts during spring and summer. SAD may be effectively treated with light therapy, but nearly half of those with SAD do not respond to light therapy alone. Antidepressant medication and psychotherapy can reduce SAD symptoms, either alone or in combination with light therapy.

Most medical research bases itself on the premise that depressive illnesses are disorders of the brain. Brain-imaging technologies, such as magnetic resonance imaging (MRI), have shown that brains of people who experience depression look different from those of people without depressive symptoms. The parts of the brain responsible for regulating mood, thinking, sleep, appetite and behaviour appear to function abnormally. But, important neurotransmitter chemicals that brain cells use to communicate appear to be out of balance.

Depression can also run in families, suggesting a genetic link, but, it can also occur in people without a family history of depression as well (NIMH, 2005). Genetic research indicates that risk of depression increases with the influence of multiple genes acting together with environmental or other factors. In addition, trauma, loss of a loved one, a difficult relationship, or any stressful situation may trigger a depressive episode. Subsequent depressive episodes may occur with or without an obvious trigger. A person with depression may be treated in a number of ways, the most common being medication and psychotherapy. Antidepressants work to normalize naturally occurring brain chemicals called neurotransmitters, notably serotonin and norepinephrine. Other 
antidepressants work on the neurotransmitter dopamine. Currently, efforts are ongoing to better understand and mitigate the various side-effects of these drugs.

There are seemingly more parallels in the use of the term depression economics and medical sciences than the use of brain terminology and economists have also spent more time and energy in both documenting and classifying depressions (see Burns and Mitchell (1946)) than analyzing brain like economic functioning. But the classifications of economic depressions used by economists seemingly bear no relationship to medical classifications. In addition, based on historical analyses, depressions and recessions are seen as episodic features of economic behaviour of all economies, and not isolated to only a few economies. Also propagation of depressions across economies is a major focus of economic research; propagation of medical depression is less discussed. Also, the wide-spread use of therapies and medication for economic agents (consumers and producers) to treat economic depressions has not, as far as we know, been suggested or analyzed by economists.

\subsection{Exhaustion in Economics and in Medical Sciences}

Another medical term finding usage in economics discussion is exhaustion. This has been used in economics literature in a variety of contexts. Hawtrey (1944) used the term to refer to economies undergoing an arms build up in which the fraction of national income devoted to military preparedness becomes so large that little by way of resources remains for conventional production and consumption. The notion is that the exhaustion of scarce resources for military or security purposes will ultimately stifle economic activity and undermine the military effort.

Another use of the term relates to exhaustion of pools of natural resources. This is analyzed by economists as cases where individual property rights over a common property resource (such as a fish stock) are not well defined. As a result, over depletion occurs to the point that the stock of the resource can not renew itself. Some believe that this same underlying problem characterizes global warming and climate change and the impact on the so called global commons due to the progressive erosion of the ability of the atmosphere for self-regulation.

Such uses of terms, however, differ quite sharply from medical uses of the same term. Fatigue and weakness are terms often interchangeably used to describe a range of stress related symptoms leading to exhaustion. These can exist in conjunction with an onset of another disease. Weakness may denote a lack of physical or muscle strength and the feeling that extra effort is needed to perform daily activities that require movement of arms, legs, or other muscles. Fatigue is a feeling of tiredness, exhaustion, or lack of energy (Green et. al., 2010).

General weakness also often occurs after an activity is conducted excessively, such as after a long hike, but in rare cases, complaints of weakness or exhaustion may be an indicator of low thyroid level (hypothyroidism) causing fatigue, weakness, lethargy, weight gain, depression, memory problems, constipation, dry skin, intolerance to cold, coarse and thinning hair, brittle nails, or a yellowish tint to the skin. Exhaustion manifests itself in both physical and mental form. Physical fatigue is the inability to continue functioning at the level of one's normal abilities. It usually becomes more noticeable during heavy exercise. Mental fatigue, on the other hand, manifests itself in somnolence (sleepiness). Exhaustion is typically the result of working, mental stress, overstimulation or under-stimulation, jet lag or active recreation, depression, and also boredom, disease and lack of sleep. It may also have chemical causes, such as poisoning or mineral or vitamin deficiencies (Green et. al., 2010).

Thus, the discussion of the term exhaustion in health science literature, like the earlier terms, is more nuanced and multifaceted compared to the use of corresponding terms in economic usage, and focuses on the ability of the human organism (unlike the economy) to regenerate itself after exhaustion rather than only what exhaustion is. Regeneration following exhaustion in economies is little discussed since exhausted resources in economic discussion are usually treated as non-renewable.

\section{Some Examples of Health Sciences Use of Economic Terminology}

We next turn to examples of health sciences use of economic terminology where a similar theme of adoption of terms from another discipline in ways that do not fully reflect their use in other disciplines emerges.

\subsection{Currency}

Our first example of the use of economic terminology in health sciences arises with ATP and it's characterization by researchers in medical science as currency, originally an economic term (Bunney et. al., 2001). ATP (Adenosine triphosphate) is a nucleoside comprised of a central ribose sugar, a purine adenine base and a chain of three phosphate groups. It is an immediate energy source in the cell and is formed in three stages. The first stage begins by harvesting chemical energy from oxidation of a glucose molecule. This process takes place in the cytoplasm and is known as glycolysis. Since the energy within organic molecules is stored within the 
individual atoms, it can only be released by breaking the bonds which hold the atoms together. This requires an 'energy spend' of two ATP molecules to assist the breakdown of glucose into intermediate substrates called glyceraldehyde-3-phosphates. Further breakdown enables the coenzyme NAD+ to pick up high-energy electrons and hydrogen ions, forming two NADH molecules. It also releases energy allowing phosphate group to bond with ADP, forming two molecules of ATP in a process called substrate level phosphorylation (McMillan, 2009).

Further breakdown to pyruvate generates an additional two molecules of ATP, giving glycolysis an overall energy 'profit' of two ATP. The next stage of cellular respiration also yields ATP by substrate level phosphorylation. This stage, known as the Citric Acid Cycle, completes the oxidation of glucose and takes place in the mitochondria of the cell. Pyruvate diffuses through the cell membrane and undergoes several chemical reactions to form Acetyl Co-A, producing carbon dioxide as a waste product. NADH and FADH2 also carry electrons during this stage as well. Another two molecules of ATP are produced which can be immediately used by the cell for energy. It is the molecular arrangement of ATP which then allows the release of this potential energy (McMillan, 2009).

It is in describing the breakdown of ATP to ADP and consequent regeneration which affords each cell that medical researchers use the term currency as needed to survive and carry out the cellular work for a particular function. The notion is that ATP is an energy currency this reflects the idea in the health sciences literature that ATP must first be 'spent' (in the form of 2 ATP molecules in the glycolysis stage) in order to gain a 'profit' of 36 ATP molecules per oxidised glucose molecule.

Since all three phosphate groups are negatively charged, the molecule is unstable and readily gives up its terminal. An exergonic reaction occurs when the molecule releases energy into its surroundings when yielding products that have less potential energy than their reactants. An endergonic reaction requires an input of energy from its surrounding and products that have more potential energy than reactants. It is the ability of ATP to couple endergonic and exergonic reactions that makes life able to continue and draws the currency labelling from health scientists. By giving up a phosphate group in the exergonic transformation of ATP to ADP, this allows other reactants to pick them up and gain energy to allow an endergonic reaction to take place. This process is known as energy coupling in the cell (McMillan, 2009).

Without continuous recycling and management of the energy in our cells by ATP, we would not have energy to make new blood, move around, rid our bodies of poisons or even reproduce. The significance of ATP was highlighted in 1997 when the Nobel Prize for Chemistry was shared between Boyer, Walker \& Skou when they consolidated the structure of ATP and the role of ATP synthase. In 1929, Karl Lohmann initially isolated ATP from muscle and liver extracts and declared it a chemical energy reservoir of the cell (Langen, 2008).

This use of the term currency, however, differs sharply from its use in economics. Currency in this use is much closer to a description of a production process involving inputs and outputs, whereas currency in economics discourse is not seen as linked to production. Rather, it is both a medium of exchange and a store of value, not an energy unit. And different currencies exist in different economies, and multiple currencies can simultaneously exist in different domestic economies (cash and credit cards for example). Currency is used to execute transactions between economic actors and the gains from trade between agents drive economies, and currencies facilitate transactions leading to these gains. Currency may thus be central to economic activity, but the elements of essentiality differ strongly from those claimed for ATP in health sciences discussion.

\subsection{Elasticity}

Another example of use of economic terminology is the term elasticity as used in health sciences literature. This use of the term arises because biological cells must meet a complex set of requirements in order to survive, function and reproduce. Red blood cells (RBC), for example, must adapt to a relatively wide range of capillary sizes found in blood vessels. They must at the same time be deformable while maintaining their cellular integrity and function. Epithelial cells that form tissues that cover the internal and external surfaces of organs (e.g., skin cells, lining of the lungs, intestines) must adhere to substrates and to each other under a wide variety of circumstances. Their adhesion properties can be regulated by the cell which simultaneously senses the chemical and mechanical properties of its environment. Fibroblasts that maintain connective tissue and play an important role in wound healing have their own internal mechanisms for regulating the elastic forces that they exert. In health sciences discussion, cell elasticity plays a vital role in all the above mentioned functions ranging from the cell membrane itself to the various organelles contained within. In all these examples, cell shape and adhesion differ from vesicle shape and adhesion due to the presence of the elastic cytoskeleton and to the fact that active processes within the cell modify cytoskeletal elasticity and tension. 
Health Sciences literature focuses on the need for elasticity which lies in the arteries surrounding the cardiovascular system (Fjeldstad, 2007). Arterial compliance is defined as the ability of an artery to expand and recoil with cardiac pulsation and relaxation. A decrease in arterial compliance or an increase in arterial stiffness is common with advancing age in both men and women and may lead to atherosclerosis regardless of the presence of coexisting diseases. Obesity may also negatively affect cardiovascular function through associations with hypertension. A growing body of evidence suggests that measures of arterial compliance (small arterial elasticity indices (SAEI) and larger arterial elasticity indices (LAEI)) relate to risk factors for coronary artery disease (CAD). Reduced compliance has been reported in hypertensive patients as well as in the diabetic patients, long-term cigarette smokers, and aging. Preliminary studies have also shown that the SAEI is inversely associated with the extent of angiographic CAD in diabetic and hypertensive subjects. Altogether, these observations have led to the use of measures of the arterial elasticity as a screening tool for the detection of coronary atherosclerosis burden.

Skin is also usually described in health sciences literature as an elastic organ of the human body Most experimental data on skin biomechanics are based on the stress-strain relationship (Berardesca, 1995). Skin does not behave as an ideal elastic object, but during continuing stress the deformation increases slowly and fails to return to its original state, as it remains slightly deformed. Instruments employing tension, torsion, suction and vibration have been developed by bioengineers to study the elasticity of the skin (e.g. a cutometer) (Berardesca, 1995).

In contrast to this use of terminology in health sciences literature, elasticity in economics is not discussed in terms of degrees of physical malleability and there is no mention of the need for elasticity. It instead characterizes the behavioural response of endogenous variables to changes in exogenous variables in economic models. Originally introduced by Alfred Marshall in the 1870's into economics, key elasticities now enter basic first year training in university economics. The price elasticity of demand captures how quantities demanded respond to changes in prices. Other elasticities are income elasticities of demand, price elasticities of supply, and elasticities of substitution. They are characterizations of behavioural response; not of the physical characteristics of bodies.

\subsection{Homeostasis and Equilibrium}

Another area of dual use of concepts in economics and health sciences arises with the closely related dual use of the concepts homeostasis and equilibrium. Homeostasis is defined in its biological context as the body's ability to maintain a constant internal environment at an optimal level regardless of changes in its external environment. There is a need for homeostasis in every living organism because it can only survive and thrive within a certain range of internal conditions. The ability of various organisms to detect even slight variations may range from temperature and $\mathrm{pH}$ of the blood to various ions and minerals required for sustenance. The lack of or any disruption to homeostasis often produces illness and even death in living organisms. Multi-cellular organisms benefit from a homeostatic mechanism since each cellular sub-type is able to protect the organism from external changes. The need for constant homeostasis is referred to as maintaining a dynamic equilibrium (Hallman, 2006).

Temperature homeostasis (thermoregulation) is one of the key regulatory functions for an organism's survival. In humans, body temperature is controlled by the thermoregulatory centre in the hypothalamus. It receives input from two sets of thermo-receptors: receptors in the hypothalamus monitor the temperature of the blood as it passes through the brain (the core temperature), and receptors in the skin (especially on the trunk) monitor the external temperature. Both sets of information are needed so that the body can make appropriate adjustments. The thermoregulatory centre sends impulses to several different effectors to adjust body temperature. Our first response to encountering a hotter or colder condition is voluntary - if too hot, we may decide to take some clothes off, or to move into the shade. It is only when these responses are not enough that the thermoregulatory centre is stimulated. This is part of the autonomic nervous system, so the various responses are all involuntary.

When we get too hot, the heat loss centre in the hypothalamus is stimulated; when we get too cold, it is the heat conservation centre of the hypothalamus which is stimulated. Some of the responses to low temperature actually generate heat (thermo-genesis), whilst others just conserve heat. Similarly some of the responses to cold actively cool the body down, while others just reduce heat production or transfer heat to the surface. The body thus has a range of responses available, depending on the internal and external temperatures.

Water and ion balance is also an integral part of the overall homeostatic balance. The body maintains a balance of water intake and output by a series of negative feedback loops involving the endocrine system and autonomic nervous system. The average adult body contains about 40 litres of water. This amount, called total body water, 
remains fairly constant under normal circumstances. We lose approximately 1500 millilitres per day from the kidneys in the form of urine. We also lose about 600 millilitres of water per day through the skin and 300 millilitres from the lungs in the form of water vapour in exhaled air (Interact Physiology, 2005). These two latter forms of water loss are called insensible loss because we are unaware of the process. It is necessary to constantly replenish the daily water requirement of the body to maintain a balance.

As such, homeostasis in medical literature stresses the role of feedback mechanisms and discusses what is needed for stability of systems in the presence of feedback interactions. Homeostasis, like equilibrium in economics, stresses balance, but unlike in economics, it is seen as something that is needed for normal bodily function. In contrast, in economics, equilibrium is discussed as the central solution concept for economic models in Newtonian mechanical terms but there is no discussion in economics of equilibrium being something that is needed. It describes an outcome in a model and disequilibrium processes are in terms of their ability to restore equilibrium. Thus, in economic equilibrium counteracting forces on the economy or on parts of a sub-economy offset each other and the economy or sub-economy is at rest. Demand equals supply in a series of specified inter-connecting markets and agent interactions and equilibrium is attained. Changes in policy or other variables shift the economy from one equilibrium to another, and comparative static analyses helps inform policy decisions. Desirable optimality properties of equilibrium then enter the analysis. There is no self-regulation mechanism that automatically restores equilibrium. Feedbacks in terms of dynamic adaptive process are discussed but little emphasized, and do not fill the central role that they occupy in medical use of homeostasis concept.

\subsection{Optimality and Optimal health}

Optimality, in the medical use, is being in the healthiest physical and mental state. This differs from one individual to another based on genetic predispositions, environmental factors, and personal health choices. It is often used in kinesiology in discussion of athletic performances when referring to one's peak or optimal performance. According to the World Health Organization, one's health is in an optimal state when one is in a "a state of complete physical, mental, and social well-being and not merely the absence of disease or infirmity"(WHO, 1948). As such medical optimality is closer to economists' notions of equilibrium than it is to economists' notions of optimality.

WHO further argue that health should be seen as a resource for everyday life, not the objective of living. Health is a positive concept emphasizing social and personal resources, as well as physical capacities. Health is thus an essential part of wellness. Wellness refers to a positive state of human flourishing that includes the physical, mental (e.g. psycho/social), and spiritual/moral dimensions of the human experience.

In contrast, in economic usage optimality characterizes the best outcome for agents or the whole economy given the constraints the economy faces. Consumers optimize by maximizing utility subject to a budget constraint, producers optimize and maximize profits. There is no notion of balance and compatibility with a wider environment in economic optimality, as characterizes personal objectives in much Eastern philosophy. Optimality in economics is simply maximizing an objective function subject to well specified constraints; it is not inherently a state of balance.

\section{Concluding Remarks and Some Implications of Economics/Health Sciences Intertwining for Discussions of Interdisciplinarity More Broadly}

Above we present some of examples of terminology usage between economics and health sciences that are inherently cross-disciplinary. Terms and concepts from one discipline are adopted modified, and applied in other. Our contention from the examples we cite between economics and health sciences is that the use of terminology in one area can differ sharply at deep conceptual levels from its use in the other (typically original) area. This if generalizable to other pair-wise disciplinary comparisons would seem to have broader implications for what may emerge from growing interdisciplinary discourse in research.

Interdisciplinary research is widely thought to be needed to address issues that are too broad or complex to be dealt with adequately by a single discipline or profession (Klein et al., 1998). According to the National Institute of Health (NIH) (Aboelela et. al., 2007) such interdisciplinarity integrates the analytical strengths of two or more often disparate scientific disciplines to solve a given problem. For instance, behavioural scientists, molecular biologists, and mathematicians might combine their research tools, approaches, and technologies to more effectively solve complex health problems such as pain and obesity. By engaging seemingly unrelated disciplines, the idea seems to be that gaps in terminology, approach, and methodology can hopefully be gradually eliminated. With roadblocks to potential collaboration removed, the hope is that a meeting of minds 
can take place that broadens the scope of investigation, yields fresh and possibly unexpected insights, and even give birth to new hybrid disciplines that are more analytically sophisticated (Aboelela et al, 2007).

However, our discussion suggests that intertwining use of concepts and terms across disciplines may have another side to it. The adoption of concepts from other disciplines in initially unrelated disciplines would seem to foster a process where adoptions can occur because of the perceived novelty injected into the receiving discipline, but in ways which either do not reflect or are partially ignorant of the original meaning. Indeed the incentive to be new within one's own discipline can engender exactly this incentive. And the fundamental different philosophical underpinnings of disciplines would seem likely to engender this outcome if one (health sciences) stresses feedbacks and balance as an organic process within a living organism and the other (economicts) stresses Newtonian mechanisms and a description of states of rest for bodies with forces acting on them. One result of such interdisciplinarity, therefore, can be a growing confusion across disciplines as to what terms mean and what the implications are for action (medical treatment or economic policy prescriptions). And to suggest that because of the 2008 financial crisis we need medical treatments for economics seems to imply cross disciplinary transfer of diagnosis and prescriptions, without acknowledging that the disciplines involved may not fully understand each other.

In this paper, we have examined only one element in interdisciplinarity; the overlapping use of language in health science and economics. Other examples of interdisciplinary cooperation involve economists teaming up with ecologists (Wam, 2010) where the two research fields are often viewed as disparate. Even though practitioners from the two fields may take different approaches when tackling similar issues such as sustainable management of natural resources, a relevant meeting arena is bio-economic modelling using bioeconomics. As an interdisciplinary methodology that combines both natural and social sciences drawing on theories in both economics and ecology in the study of biological resource dynamics (Wam, 2010), it would also be interesting to know how far the use and interpretation of concepts are divergent.

Karl Popper noted: 'We are not students of some subject matter, but students of problems. And problems may cut right across the borders of any subject matter or discipline.' Interdisciplinary research is inspired by the drive to answer complex questions and encourage researchers to meet at the frontiers of current disciplines and cross them to form new ones. This trend in natural sciences has lead to several hybrid fields such as bioengineering and biogeochemistry. Challenges that need to be faced in expanding such research are multiple. Since researchers are often from disparate backgrounds, it requires time for bridging differences in language, culture, and new methods in order to build consensus. Academic institutions thus need not to only to develop and strengthen current policies that lower or remove barriers to interdisciplinary scholarship but to also focus on the large philosophical differences that are at the root of interdisciplinary boundaries.

Collaboration between professional institutions and government educational initiatives can also help create greater interdisciplinarity between research fields even from undergraduate levels and lead to a better transition to advanced interdisciplinary research but it would be greatly added by the disciplines involved first attempting to better understand each other before interdisciplinarity proceeds. Some academic cynics often view interdisciplinarity as in part, an inconvenience imposed on them by research councils in their pursuit of sound engagement of researchers for the greater good. Even though tensions over budgets, space and intellectual underpinnings may result in the short run from interdisciplinarity, the opportunity for positive outcomes via such collaboration is far more significant. We suggest that mutual understanding of the disciplines to be interdisciplinized may be useful precursor.

\section{Acknowledgements}

We are grateful to the Centre for the Study of International Economic Relations (CSIER) in Department of Economics at Western University for financial support and to Richard Higgott for helpful discussions.

\section{References}

Aboelela, S. W., Larson, E., Bakken, S., Carrasquillo, O., Formicola, A., Glied, S., Haas, J., \& Gebbie K. M. (2007). Defining Interdisciplinary Research: Conclusions from a Critical Review of the Literature. Health Services Research, 42(1 Pt 1), 329-346. http://dx.doi.org/10.1111/j.1475-6773.2006.00621.x

Berardesca, E. (1995). Bioengineering of the skin: methods and instrumentation. Florence, KY: CRC Press.

Bunney, T. D., Hendrika S. van Walraven, \& Albertus H. de Boer. (2001). 14-3-3 protein is a regulator of the mitochondrial and chloroplast ATP synthase. Proc Natl Acad Sci U S A., 98(7), 4249-4254. d http://dx.doi.org/10.1073/pnas.061437498 
Fjeldstad, A. S., \& Bemben, D. (2007). Large and small arterial elasticity in healthy active and sedentary premenopausal women. Journal of Sports Science and Medicine, 6, 250-253. Retrieved from http://www.jssm.org/vol6/n2/13/v6n2-13pdf.pdf

Green, W. M. et. al. (2010). Weakness And Fatigue. Healthwise. Retrieved from http://www.aolhealth.com/symptom/weakness-and-fatigue

Hallman, R. (2006). Reviewing the living environment. New York, NY: Amsco School Pubns Inc. Revised edition (March 31, 2006).

Hawtrey, R. G. (1999). Economic destiny. London UK: Longmans, Green and Co.

Hoyle, B., \& Arthur, P. (2005). Central Nervous System. Gale Encyclopaedia of Neurological Disorders. Retrieved from http://www.encyclopedia.com/doc/1G2-3435200081.html

Burns, A. F., \& Mitchell, W. C. (1946). Measuring Business Cycles. New York: National Bureau of Economic Research.

Interactive Physiology. (2005). Water Homeostasis. Retrieved from http://www.winona.edu/biology/adam_ip/misc/assignmentfiles/fluidsandelectrolytes/Water_Homeostasis.pdf

Klein, J. T., \& William, H. N. "Advancing Interdisciplinary Studies." Pp. 3-22 in Interdisciplinarity: Essays from the Literature, William H. Newell (ed). New York: College Entrance Examination Board, 1998: 3.

Krugman, P. (2009). The Return of Depression Economics and the Crisis of 2008, New York, NY: W. W. Norton \& Company.

Langen, P., \& Hucho, F. (2008). Karl Lohmann and the Discovery of ATP. Angew. Chem. Int. Ed., 47: 1824-1827. http://dx.doi.org/10.1002/anie.200702929

McMillan, K. (2009). ATP Structure, Formation and Function-The Energy Currency of Cellular Metabolism. Retrieved from http://ezinearticles.com/?ATP-Structure,-Formation-and-Function---The-Energy-Currency-ofCellular-Metabolism\&id=2275923

National Institute of Mental Health. (2008). Depression. Retrieved from http://www.nimh.nih.gov/health/publications/depression/depression-booklet.pdf

Popper, K. R. (1963). Conjectures and Refutations: The Growth of Scientific Knowledge. New York: Routledge and Kegan Paul.

Stiglitz, J. (2008). Falling Down. Retrieved from http://www.tnr.com/article/falling-down?id=947bf9e5-923b409a-adac-579658c99ddf

Wam, H. K. (2010). Economists, time to team up with the ecologists! Ecological Economics, 69(4), 675-679. http://dx.doi.org/10.1016/j.ecolecon.2009.12.005

World Health Organization. (1948). WHO definition of Health. Retrieved from http://who.int/about/definition/en/print.html

\section{(cc) BY}

This work is licensed under a Creative Commons Attribution 3.0 License. 